\title{
SISTEMATIZAÇĀO DA ASSISTÊNCIA DE ENFERMAGEM AOS PACIENTES SUBMETIDOS À REMOÇÃO POR MEIO DE TRANSPORTE AÉREO
}

\author{
Rosana Chami Gentil*
}

GENTIL, R.C. Sistematização da assistência de enfermagem aos pacientes submetidos à remoção por meio de transporte aéreo: nota prévia. Rev. Esc. Enf. USP, São Paulo, 23(2): - , ago. 1989.

\section{NOTA PRÉVIA.}

No desempenho de nossas atividacies protissionais, observamos que a remoção de pacientes em grau variável de gravidade, feita por transporte aéreo, tem ocorrido com maior frequiência nos últimos anos, seja na presteza do transporte de pacientes por solicitação familiar, como para esclarecimento diagnóstico em hospitais de maiores recursos.

A verdade é que ela é um fato e a assistência de enfermagem se faz necessária nessa ocasião.

Oosservamos outrossim, que a assistência de enfermagem utilizada nessa situação, parece ocorrer sem planejamento, sem uma sistematizaçāo, embora seja evidente sua importância.

Em nosso meio a literatura sobre o assunto é muito escassa, e despertou nosso interesse no sentido de desenvolver um instrumento que sistematize a assistência de enfermagem, a pacientes submetidos a esse tipo de serviço.

GENTIL, R.C. Nurse care systematization for airplane transportation of critically ill patients: preliminary note. Rev. Esc. Enf. USP, São Paulo, 23(2) -, Aug. 1989.

\section{PRELIMINARY NOTE}

In last few years there are a increasing frequency of patients who need air. lanes trasportation, either by familiar seeking or to diagnosis estabilishment in a tertiary care center.

Although the need of specially trained nurse care it does not seem to occur in our country.

We are concerned to perform a systematization in airplane transportation of critically ill patients because there are no reports on it.

\footnotetext{
Recebido para publicação em 25/08/88.
}

\footnotetext{
* Enfermeira Supervisora da Unidade de Primeiro Atendimento do Hospital Albert Einstein - Pos-Graduanda em Administração Hospitalar da Faculdade de Ciências São Camilo.
} 